\title{
Conversion of hulled into naked barley by Cas endonuclease-mediated knockout of the NUD gene
}

Sophia V. Gerasimova ${ }^{1,2,3^{*}}$ (D), Christian Hertig ${ }^{4 \dagger}$, Anna M. Korotkova ${ }^{1}$, Ekaterina V. Kolosovskaya ${ }^{1}$, Ingrid Otto ${ }^{4}$, Stefan Hiekel ${ }^{4}$, Alex V. Kochetov ${ }^{1,2}$, Elena K. Khlestkina ${ }^{1,2,5 \dagger}$ and Jochen Kumlehn ${ }^{4 \dagger}$

From Fifth International Scientific Conference "Plant Genetics, Genomics, Bioinformatics, and Biotechnology" (PlantGen2019) Novosibirsk, Russia. 24-29 June 2019

\begin{abstract}
Background: The naked caryopsis character in barley is a domestication-associated trait defined by loss-of-function of the NUD gene. The functional NUD gene encodes an Apetala 2/Ethylene-Response Factor (AP2/ERF) controlling the formation of a cementing layer between pericarp and both lemma and palea. The downstream genes regulated by the NUD transcription factor and molecular mechanism of a cementing layer formation are still not sufficiently described. A naturally occurring 17-kb deletion in the nud locus is associated with the emergence of naked barley. Naked barley has been traditionally used for food and nowadays is considered as a dietary component for functional nutrition.

Results: In the present study, we demonstrate that targeted knockout of the NUD gene using RNA-guided Cas9 endonuclease leads to the phenotype conversion from hulled to naked barley. Using in vivo pre-testing systems, highly effective guide RNAs targeting the first exon of the NUD gene were selected. Expression cassettes harboring the cas 9 and guide RNA genes were used to transform barley cv. Golden Promise via Agrobacterium-mediated DNA transfer. The recessive naked grain phenotype was observed in $57 \%$ of primary transformants, which indicates a frequent occurrence of homozygous or biallelic mutations. T-DNA-free homozygous lines with independently generated mutations in the NUD gene were obtained in the T1 generation. At homozygous state, all obtained mutations including one- and two-amino acid losses with the translational reading frame being retained invariably caused the naked grain phenotype.

(Continued on next page)
\end{abstract}

\footnotetext{
*Correspondence: gerson@bionet.nsc.ru; sonya.gerasimova@gmail.com

†Sophia V. Gerasimova, Christian Hertig, Elena K. Khlestkina and Jochen Kumlehn contributed equally to this work.

'Institute of Cytology and Genetics, SB RAS, Novosibirsk 630090, Russia

${ }^{2}$ Novosibirsk State University, Novosibirsk 630090, Russia

Full list of author information is available at the end of the article
}

(c) The Author(s). 2020 Open Access This article is licensed under a Creative Commons Attribution 4.0 International License, which permits use, sharing, adaptation, distribution and reproduction in any medium or format, as long as you give appropriate credit to the original author(s) and the source, provide a link to the Creative Commons licence, and indicate if changes were made. The images or other third party material in this article are included in the article's Creative Commons. licence, unless indicated otherwise in a credit line to the material. If material is not included in the article's Creative Commons licence and your intended use is not permitted by statutory regulation or exceeds the permitted use, you will need to obtain permission directly from the copyright holder. To view a copy of this licence, visit http://creativecommons.org/licenses/by/4.0/ The Creative Commons Public Domain Dedication waiver (http://creativecommons.org/publicdomain/zero/1.0/) applies to the data made available in this article, unless otherwise stated in a credit line to the data. 
(Continued from previous page)

Conclusions: The hulled and naked barley isogenic lines generated are a perfect experimental model for further studies on pleiotropic consequences of nud mutations on overall plant performance under particular consideration of yield-determining traits. Due to the high $\beta$-glucan content of its grains, naked barley is considered as being of particular dietary value. The possibility to convert hulled into naked barley cultivars by targeted mutagenesis allows breeders to extend the potential utilization of barley by the provision of functional food.

Keywords: Hordeum vulgare, Naked barley, Cas9, Gene knockout, Domestication, Protoplasts, Targeted mutagenesis

\section{Background}

One of the most clearly distinguishable traits of barley which emerged during the process of domestication is the naked versus the hulled caryopsis character. Barley cultivation is dated back to about 10,000 years before present day [1]. The naked barley trait occurred early during domestication, that is, already in the 7th millennium B.C. It appeared in different combinations along with other characteristics, such as various grain colors and two- or six-rowed spikes [2]. While barley domestication is well established as being of multiple independent origin, naked grain barley is usually considered to be monophyletic [3, 4]. The vast majority of naked barley accessions are harboring a $17-\mathrm{kb}$ deletion at the same locus located on the long arm of chromosome $7 \mathrm{H}$ [4-7]. The locus is called NUD (for nudum) and in hulled varieties, it contains a gene (NUD) encoding an Ethylene Response Factor (ERF) family transcription factor belonging to the group of Wax Inducer $1 /$ Shine 1 (WIN1/ SHN1)-like transcription factors [5]. Re-sequencing of the NUD locus in large panels of barley varieties showed the intact NUD gene in all hulled accessions, whereas all naked ones featured either the aforementioned 17-kb deletion or a T643A amino acid conversion [6]. An analysis of X-ray-induced naked grain mutants confirmed the expectation that the NUD gene carried nonsynonymous single nucleotide polymorphisms in all cases [5]. More recently, targeted mutagenesis of the NUD gene was also demonstrated to cause the appearance of naked grains on primary transgenic plants [8].

Genotypes with naked grains were selected and fixed during domestication of the majority of cereal crops, but the origin of this trait is not uniform. The naked grain of bread wheat is associated with soft glumes in contrast to tough glumes of wild progenitors [9]. The mechanism of hulled grain formation in barley is apparently different from wheat in that it relies on the occurrence of a lipid-based cementing layer between pericarp and hull $[5,10]$. The exact mechanism of cementing layer formation and the role of the NUD gene in this process are still not well understood. The NUD gene was found to be expressed during grain development in the integument that constitutes the coat of the ovule, while the cementing layer occurs at the surface of the pericarpal epidermis [5]. There are no other genes known to be involved in hulled barley control. The recent genome-wide association analysis on 525 spring barley landraces corroborated the key role of the NUD gene in naked grain formation [7]. A comparative transcriptome analysis of hulled and hulless barley accessions revealed a suppression of genes involved in pericarpal cuticle organization in hulled barley. It was hypothesized that the NUD gene may be a master regulator of these genes to increase cuticle permeability thereby causing the hull-caryopsis fusion [11]. However, under consideration of the different genetic backgrounds of the compared germplasm, the observed differences could not be directly related to the NUD gene. Consequently, thus far available data do not allow for an elucidation of the molecular mechanisms of cementing layer formation in hulled barley.

The use of customizable endonucleases allows one to induce desirable modifications at defined genomic loci [12]. With the emergence of RNA-guided Cas endonucleases, the number of examples of site-directed genome modification has been strongly increasing in crop plants [13]. The technology is currently well established in barley, including both reverse genetics studies and improvement of agronomically important characteristics [14].

The present study aims to strengthen the body of evidence for the causal effect of loss-of-function of the NUD gene on the formation of non-adherent hulls in barley. This is demonstrated by Cas endonucleasemediated generation and comparison of isogenic lines carrying either functional or non-functional alleles of the NUD gene in the context of an identical, transgene-free genetic background. A further goal is the generation of such perfectly isogenic lines which will facilitate future studies on anticipated pleiotropic effects of the naked barley character on qualitative and quantitative plant features. And finally, the conversion of hulled into naked barley without any linkage drag shall provide breeders with the unprecedented opportunity of readily introducing performance-related features of the most advanced hulled barley germplasm to naked barley.

\section{Results}

\section{Targeted knockout of the NUD gene}

Four different target motifs were selected within the Apetala 2 domain-encoding region of the NUD gene 
(Fig. 1a). For these sites, guide RNAs (gRNAs) were designed (Additional file 1, Table S1) and incorporated in the pSH121 generic vector [15].

Two different in vivo test systems were applied to select the appropriate gRNAs for stable plant transformation. For the preliminary selection of gRNAs, a previously described test system based on reporter gene restoration upon targeted mutagenesis of test constructs was used [16]. Co-bombardment of barley epidermis with pairs of a cas9/gRNA and a corresponding TARGET vector followed by quantification of mutagenesis efficacy showed that all designed gRNAs were capable of inducing mutations in their cognate target motifs in vivo. The activity, being measured as the ratio of cells showing reporter restoration per total transgeneexpressing cells, ranged from 0.27 to 0.53 (Additional file 1, Table S1). Based upon these results, it was decided to select two gRNAs, namely Nud45 and Nud50, for further experiments. Both corresponding target motifs are located within the first exon of the NUD gene in a highly conserved region of the AP2-domain (Fig. 1a), which provides the highest probability to induce loss-offunction mutations. To reveal the in situ mutation frequencies and patterns induced by the preselected gRNAs in the NUD gene, an assay was utilized, that relies on transient expression in protoplasts. To this end, transformation vectors carrying the Nud45 and Nud50 gRNAs were introduced into barley mesophyll protoplasts through polyethylene glycol-mediated transfection. Genomic DNA extracted from transformed protoplasts was used to determine the efficiency of target-specific mutagenesis. After normalization to GFP co-transformation efficiency, deep-sequencing of amplicons from the target regions revealed high portions of mutated reads for both cas $9 /$ gRNA vectors tested; $100 \%$ for Nud 45 and $71 \%$ for
Nud50 (Additional files 2 and 3, Tables S2, S3). The frequency and pattern of mutations induced within the target motifs appeared to be significantly different $(p<0,01)$ between two gRNAs, according to the two-sample Kolmogorov-Smirnov test. The distribution of mutation types obtained is presented in Fig. 2.

The Nud45 gRNA showed the highest activity in both preliminary tests. The off-target analysis revealed four predicted identical target motifs for the Nud45 gRNA in the barley genome which are located at different genomic loci including the NUD gene. Being located close to the Nud45 target motif, the on-target of the Nud50 gRNA also has three entirely matching 20-nt off-target counterparts in the genome, but none of these is followed by NGG, that is, the protospacer-adjacent motif (PAM) required for Cas9 to bind and process the target (Fig. 1b). Both of the selected gRNAs were used for stable plant transformation, which enabled us to compare their activity between transient expression assay and stably transformed plants as well as to evaluate the off-target effects in perfectly matching off-target loci with versus without functional PAM.

Next, cas 9 and gRNA expression units were transferred from the plasmids used for the protoplast assay into binary vectors for Agrobacterium-mediated plant transformation (Fig. 3a). After DNA transfer to immature embryos, 46 independent primary transgenic plants (T0) were obtained for the Nud45 gRNA and 48 transformants for the Nud50 gRNA. The analysis of Sanger sequencing chromatograms of leaf samples taken from individual T0 plants (Fig. 3b) revealed that 35 plants carried mutations in the Nud45 and 20 in the Nud50 target. The detected mutations were either homozygous (where no wild-type and no more than one mutant allele was found), heterozygous (where a single mutation was

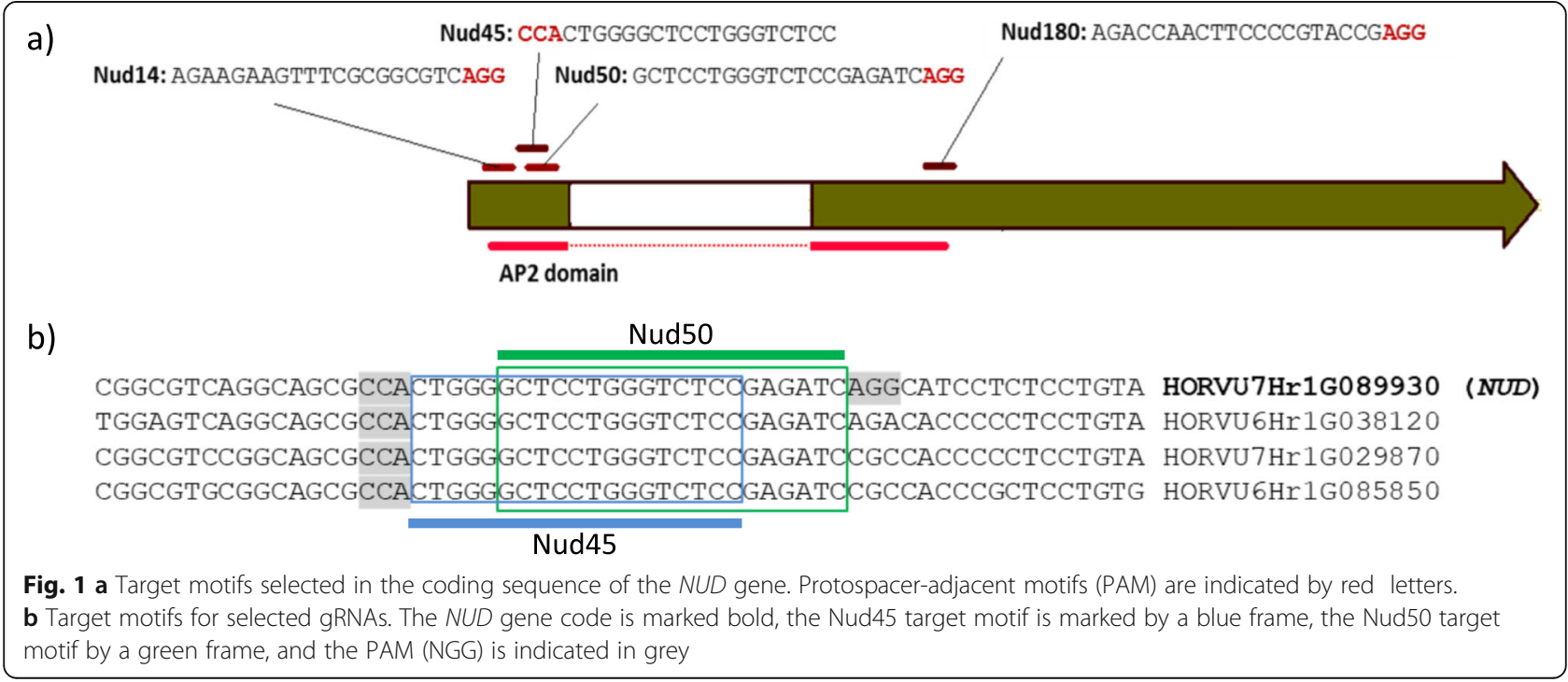




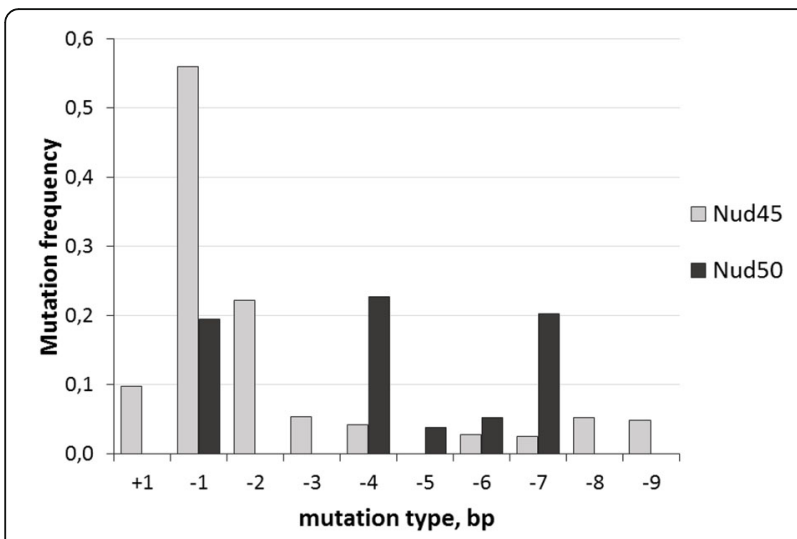

Fig. 2 Frequency and pattern of target-specific mutations in transfected protoplasts combined with the wild-type sequence), or biallelic (where two different mutant alleles were present). Of note, heterozygous and biallelic mutants cannot be discriminated from chimeric ones which carry different sectors with wild-type and/or various mutant alleles. Also owing to possible chimerism, the leaf samples taken were not necessarily representative for the whole plants. The leaf samples taken from $8 \mathrm{Nud} 45$ primary transgenic plants did not contain more than one mutant NUD allele each; 5 plants carried a 1 bp deletion, 1 plant a $3 \mathrm{bp}$ deletion, 1 plant a 1 bp insertion and 1 plant a $\sim 200$ bp deletion combined with a $5 \mathrm{bp}$ insertion. All detected mutations in Nud50 primary mutants were in heterozygous or biallelic state (Fig. 3c). However, only few analyzed plants produced naked grains (Fig. 3d), others formed hulled or both hulled and naked caryopses, suggesting a sectorial presence of wild-type, homozygous, biallelic and heterozygous alleles in chimeric primary mutant plants (Table 1).

a)

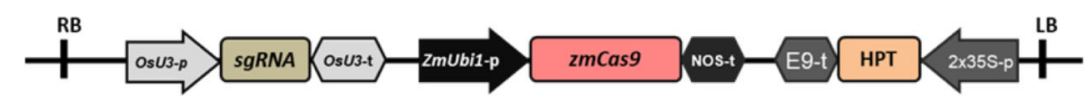

b)

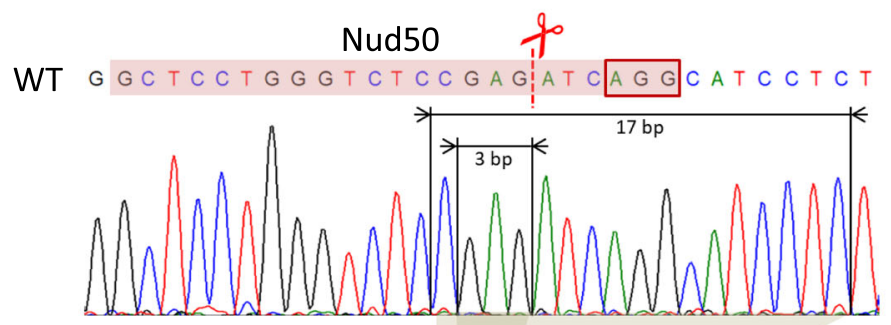

Plant 16 G G C T C C T G G G T C T C

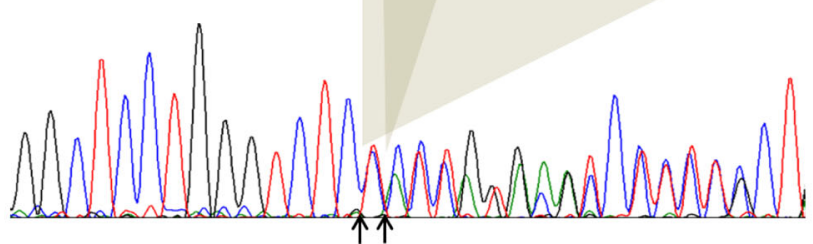

C)

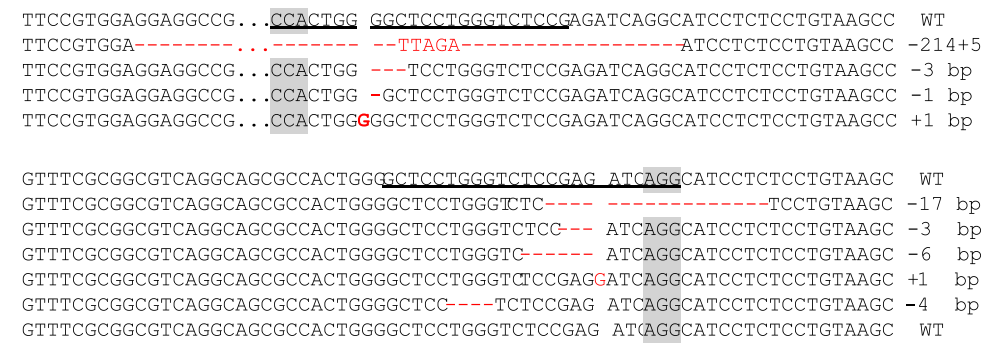

d)

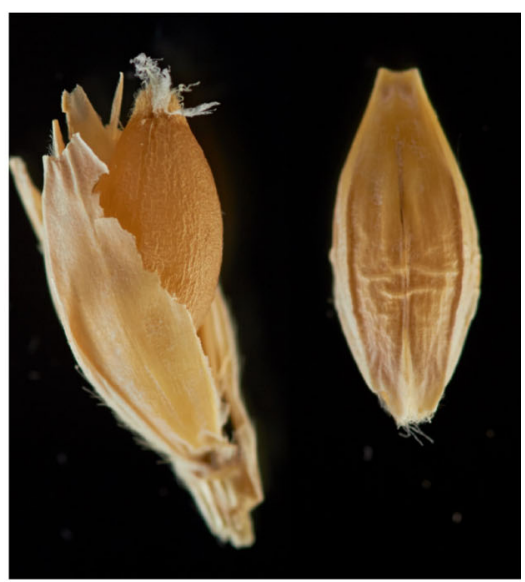

Nud45 target motif

Nud45 Plant 04

Nud45 Plant 14

Nud45 Plant 27

Nud45 Plant 41

Nud50 target motif

Nud50 Plant 16 allele 1

Nud50 Plant 16 allele 2

Nud50 Plant 31 allele 1

Nud50 Plant 31 allele 2

Nud50 Plant 33 allele 1

Nud50 Plant 33 allele 2

Fig. 3 a Binary vector T-DNA architecture, OsU3-p - rice U3 promoter; gRNA - chimeric guide RNA; OsU3-t - rice U3 terminator; ZmUbi1-p maize Ubiquitin 1 promoter; zCas9 - maize codon-optimized Streptococcus pyogenes cas 9 endonuclease gene; Nos-t - 3'-signal of Agrobacterium tumefaciens nopaline synthase gene; E9- $\mathrm{t}$ - pea Ribulose-1,5-Bisphosphate Carboxylase Small Subunit (rbcS) E9; HPT - hygromycin phosphotransferase, 2x35S-p - doubled enhanced CaMV 35 S promoter. b Mutation detection in T0, Sanger chromatogram of intact (WT) and mutated (plant 16) Nud50 target motif. The sequence with colored background indicates the target motif with the PAM being also marked by a red frame. Arrows indicate fragments deleted from the WT sequence and the corresponding ligation points in the mutant alleles of the biallelic plant 16. c Examples of mutated alleles of the NUD gene found in T0 population after mutagenesis directed by the Nud45 and Nud50 gRNAs. Target motifs are underlined, the PAM is marked grey. $\mathbf{d}$ Phenotype of grain harvested from mutant plant (left) in comparison to wild-type 'Golden Promise' grain (right) 
Table 1 Analysis of leaf samples taken from T0 plants with cas9/gRNA expression units being stably integrated

\begin{tabular}{|c|c|c|c|c|c|c|c|c|}
\hline \multirow[t]{2}{*}{ gRNA } & \multicolumn{4}{|l|}{ Genotype } & \multicolumn{4}{|l|}{ Phenotype } \\
\hline & $\begin{array}{l}\text { transgenic T0 } \\
\text { plants }\end{array}$ & $\begin{array}{l}\text { mutated } \\
\text { plants }\end{array}$ & $\begin{array}{l}\text { homozygous } \\
\text { mutations }\end{array}$ & $\begin{array}{l}\text { Efficiency } \\
(\%)\end{array}$ & $\begin{array}{l}\text { Plants with } \\
\text { grains }\end{array}$ & $\begin{array}{l}\text { only hulled } \\
\text { grains }\end{array}$ & $\begin{array}{l}\text { hulled and naked } \\
\text { grains }\end{array}$ & $\begin{array}{l}\text { only naked } \\
\text { grains }\end{array}$ \\
\hline Nud45 & 46 & 35 & 8 & 76 & 40 & 7 & 27 & 6 \\
\hline Nud50 & 48 & 20 & 0 & 42 & 43 & 22 & 16 & 5 \\
\hline
\end{tabular}

The off-target analysis was performed only for T0 mutants with detected homozygous mutations. Seven T0 plants from the Nud45 mutant population harboring different types of homozygous mutations in the NUD gene were checked for the presence of mutations in the three perfectly matching off-target loci. As a result, mutations were detected at all three off-target loci in every ontarget mutant plant.

\section{Generation of T-DNA-free knockout lines}

In order to develop T-DNA-free mutant lines, three plants having different mutation types were selected from the Nud45 and Nud50 T0 populations for further propagation and analysis (Fig. 3c, Additional file 4, Table S4). Five T1 grains (grown on T0 plants) were planted per selected primary Nud45 mutant and 30 T1 grains per Nud50 T0 mutant.

In the Nud45 T1 families, two transgene-free plants having homozygous mutations in the NUD gene were identified. Both of these, however, also carried homozygous mutations in all three perfectly matching off-target loci. All other T1 plants from this population had inherited the cas 9 and gRNA transgenes along with mutations in all four investigated target loci. In the Nud50 T1 families, transgene-free segregants each harboring one of the following homozygous mutations in the NUD gene were identified: $1 \mathrm{bp}$ insertion and three deletions of different length $(-1,-3$ and $-17 \mathrm{bp})$. In addition, two further types of homozygous deletions ( -6 and $-19 \mathrm{bp}$ ) were identified among those Nud50 T1 segregants that still proved transgenic. Among the different mutation patterns found at the on-target sites in both mutant populations, three types of in-frame mutations were identified; single amino acid deletions $\Delta \mathrm{G} 17$ and $\Delta \mathrm{E} 22$ as well as a deletion of two amino acids in positions 21 and $22(\Delta \mathrm{SE} 21 / 22)$. The mutations obtained in $\mathrm{T} 1$ are shown in Table 2 and Fig. 4. A summary of NUD gene mutation types detected in selected primary transgenic plants and corresponding T1 progeny is given in Additional file 4 (Table S4). The naked caryopsis phenotype was present in all plants harboring homozygous mutations in the NUD gene including those mutations with the reading frame being retained. Very few individual grains of NUD $\mathrm{KO}$ homozygous plants remained with hulls after standard mechanical threshing. Yet, their hulls could readily be removed manually. By contrast, the wild-type $N U D$ allele in progeny of primary transgenic plants was invariably associated with the formation of hulled grains, i.e. the hulls were neither removable by mechanical threshing nor by hand in all registered cases (Fig. 5).

In addition to the three perfectly matching Nud45 offtargets, there are three aforementioned potential offtarget sites in the barley genome which are perfectly coinciding with the target-specific part of the Nud50 gRNA, while not having an appropriate PAM site. In all on-target T1 mutants, no mutations were detected whatsoever in these PAM-less off-targets.

\section{Discussion}

\section{Mutations induced in the NUD gene}

The target site-specific activity of gRNA-navigated Cas9 and the resultant mutation patterns are highly variable. The validation of gRNA activity in vivo prior to whole-

Table 2 Types of homozygous mutations identified in the T1 generation

\begin{tabular}{lllll}
\hline gRNA & Type of mutation & No. of plants & No. of transgenic plants / No. of transgene-free plants & NUD CDS reading frame \\
\hline Nud45 & $-1 \mathrm{bp}$ & 2 & $2 / 0$ & Shifted \\
& $-3 \mathrm{bp}$ & 2 & $2 / 0$ & Retained \\
& $-214 /+5 \mathrm{bp}$ & 2 & $0 / 2$ & Shifted \\
Nud50 & $-1 \mathrm{bp}$ & 8 & $6 / 2$ & Shifted \\
& $-3 \mathrm{bp}$ & 6 & $4 / 2$ & Retained \\
& $-6 \mathrm{bp}$ & 5 & $5 / 0$ & Retained \\
& $-17 \mathrm{bp}$ & 8 & $1 / 2$ & Shifted \\
& $-19 \mathrm{bp}$ & 1 & $0 / 1$ & Shifted \\
& $+1 \mathrm{bp}(\mathrm{G})$ & 1 & & Shifted \\
\hline
\end{tabular}


a) Mutations in T1 Nud45 population

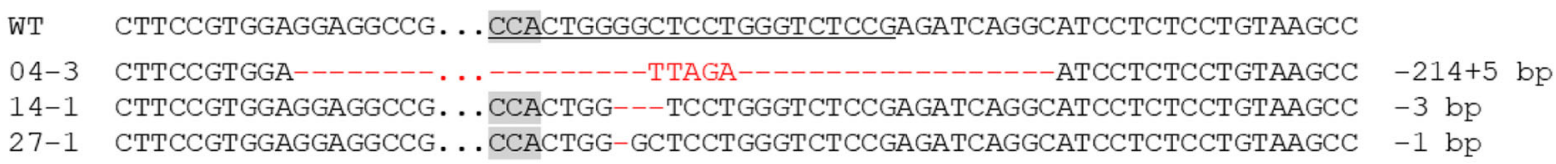

Mutations in T1 Nud50 population

WT GTTTCGCGGCGTCAGGCAGCGCCACTGGGGCTCCTGGGTCTCCGAG ATCAGGCATCCTCTCCTGTAAGC 16-6 GTTTCGCGGCGTCAGGCAGCGCCACTGGGGCTCCTGGGT-_-_-_16-14 GTTTCGCGGCGTCAGGCAGCGCCACTGGGGCTCCTGGGTCTCC--- ATCAGGCATCCTCTCCTGTAAGC 31-12 GTTTCGCGGCGTCAGGCAGCGCCACTGGGGCTCCTGGGTC------ ATCAGGCATCCTCTCCTGTAAGC 31-19 GTTTCGCGGCGTCAGGCAGCGCCACTGGGGCTCCTGGGTCTCCGAGGATCAGGCATCCTCTCCTGTAAGC 33-10 GTTTCGCGGCGTCAGGCAGCGCCACTGGGGCTCCTGGGTCTCCGA- ATCAGGCATCCTCTCCTGTAAGC 33-19 GTTTCGCGGCGTCAGGCAGCGCCACTGGGGCTCCTGGG-_-_-_-

$-17 \mathrm{bp}$ $-3 \mathrm{bp}$ $-6 \mathrm{bp}$ $+1 \mathrm{bp}(\mathrm{G})$

$-1 \mathrm{bp}$ -19 bp

b) Changes in protein primary structure

$\begin{array}{llcll} & \text { MVQSKKKFRGVRQRHWGSWVSEIRHPLL } & \text { WT } & \\ 04-3 & \text { No product } & -214+5 \mathrm{bp} & \text { Nud45 } \\ 14-1 & \text { MVQSKKKFRGVRQRHW-SWVSEIRHPLL } & -3 \mathrm{bp} & \text { Nud45 } \\ 27-1 & \text { MVQSKKKFRGVRQRHWAPGSPRSGILSC } & -1 \mathrm{bp} & \text { Nud45 } \\ 16-6 & \text { MVQSKKKFRGVRQRHWGSWVS----PVS } & -17 \mathrm{bp} & \text { Nud50 } \\ 16-14 & \text { MVQSKKKFRGVRQRHWGSWVS-IRHPLL } & -3 \mathrm{bp} & \text { Nud50 } \\ 31-12 & \text { MVQSKKKFRGVRQRHWGSWV--IRHPLL } & -6 \mathrm{bp} & \text { Nud50 } \\ 31-19 & \text { MVQSKKKFRGVRQRHWGSWVSEDQASSP } & +1 \mathrm{bp} & \text { Nud50 } \\ 33-10 & \text { MVQSKKKFRGVRQRHWGSWVSESGILSC } & -1 \mathrm{bp} & \text { Nud50 } \\ 33-19 & \text { MVQSKKKFRGVRQRHWGSWVS-----CK } & -19 \mathrm{bp} & \text { Nud50 }\end{array}$

Fig. 4 Cas9/gRNA-induced homozygous mutations in the NUD gene found in the T1 mutant population. Numbers correspond to individual plants. a Alterations in the nucleotide sequence, the PAM is marked grey. $\mathbf{b}$ Alterations in the amino acid sequence of the first exon. The DNAbinding motif is marked grey

plant genome modification allows one to achieve a high portion of plants with specific alterations in the target motif [17]. Here, two different test systems were used to select efficient guide RNAs for targeted mutagenesis of the NUD gene in barley. The results of preliminary tests were in good agreement with mutation frequency and pattern in transgenic plants and their progeny. In this study, different mutation patterns were seen in the two addressed target motifs nud 45 and nud50. In the protoplast system, the predominantly occurring mutation induced by the Nud45 gRNA was a one-nucleotide deletion. This proved also to hold true for the T0 plants carrying the same gRNA, where 5 out of 8 homozygous mutants had the very same modification. By contrast, the Nud50 target motif showed more diverse mutation types both in protoplasts and mutant plants. There was a high portion of T0 mutants having both alleles mutated in different, that is biallelic, manner, whereas no homozygous mutants were obtained in these cases. The achieved mutation frequencies for the Nud45 target motif were higher than for the Nud50 target motif in all experiments. In T0 plants, the corresponding frequencies were as high as 76 and $42 \%$, respectively. This was sufficient to obtain the desired recessive phenotype at reasonably high proportion already among T0 plants, from which transgene-free homozygous T1 mutants also efficiently segregated. The high mutation frequency was comparable with results previously reported by Kapusi et al. [18], Holubova et al. [19] and $\mathrm{Li}$ et al. [20], where similar principles of target motif selection and gRNA prevalidation had been implemented to knockout the barley ENGase, CKX1 and Albostrians genes, respectively.

Two gRNAs used in this study have three perfectly matching 20-nt off-target loci, but in case of the Nud45 gRNA, off-targets are associated with a functional PAM, whereas adjacent to the Nud50 gRNA off-targets there is no appropriate PAM. It is demonstrated that a Nud45 gRNA with high predicted activity is capable of simultaneously inducing mutations at all four different genomic target loci. This opens the opportunity to efficiently knockout gene families using just one or few highly efficient gRNA(s). The Nud45 off-targets are located in $A P 2 / E R F$ genes with unknown functions, which are sharing sequence homology with the NUD gene. Multiple mutant plants obtained in this study can be further used for reverse genetic studies of those NUDrelated genes' function. 
a)

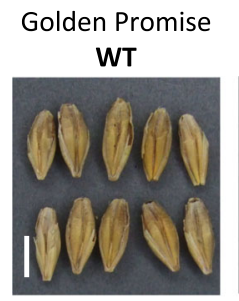

line 16-14

$-3 \mathrm{bp}$

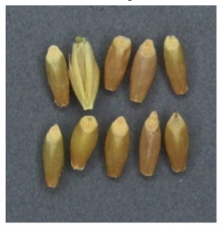

line 31-12

line 33-10

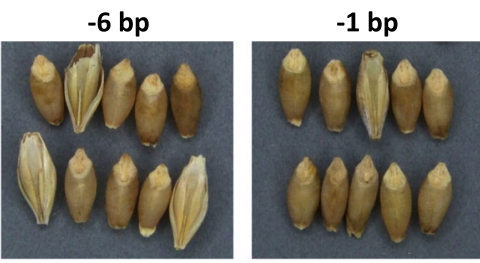

b)
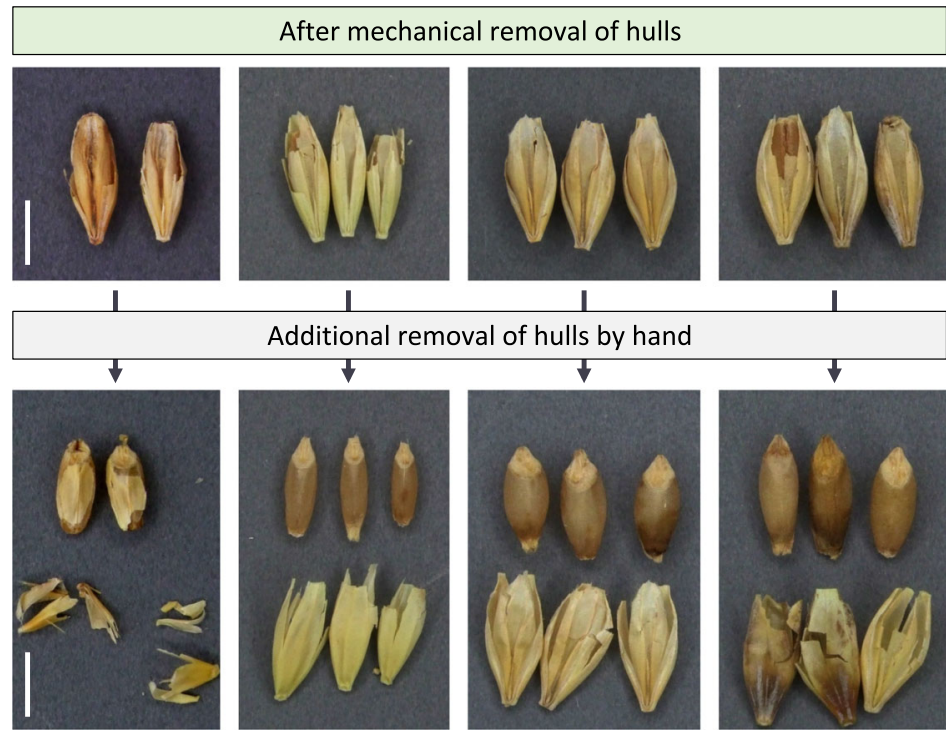

Additional removal of hulls by hand
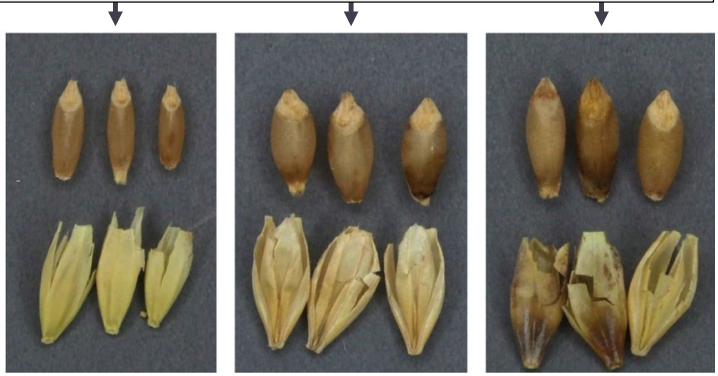

Fig. 5 T2 grains collected from NUD homozygous knockout Nud50 T1 mutants. a Grain samples collected from individual plants from three independent mutant lines (No. 16, 31 and 33) after mechanical threshing. b Manual removal of hulls that remained attached to grains after the standard threshing procedure. White bars represent $5 \mathrm{~mm}$

\section{Different mutations in the NUD gene lead to the naked grain phenotype}

The occurrence of naked grains has previously been observed in primary transgenic barley plants bearing cas 9 and NUD gene-specific gRNA expression units [8]. While leaf samples were reported to have homozygous, biallelic or heterozygous/chimeric mutation patterns, there was however no clear association of individual genotypes with phenotypes at the whole-plant level demonstrated. By contrast, in the present study, various nud gene mutations detected in T0 plants were shown to be transmitted to transgene-free progeny that were also confirmed not to carry any off-target mutations. Therefore, it was possible to unambiguously assign the naked grain phenotype to homozyogus nud mutant segregants. These $\mathrm{T} 1$ plants themselves gave rise to offspring invariably carrying naked grains. Not only frameshift mutations but also in-frame mutations associated with the loss of one or two amino acids in the AP2 domain of the NUD gene caused the conversion of hulled into naked barley. Both selected target motifs are located within the first exon at the $5^{\prime}$ part of the AP2 domain. The obtained one- and two-amino acid deletions appear within the nucleotide binding site (Fig. 4b), that is in a highly conserved region. The loss of function associated with these allelic variants, as demonstrated in the present study, provides convergent evidence of the essential role of this domain for the NUD transcription factor.

The hulled caryopsis character is defined by the NUD gene expressed in the coat of the ovule thereby regulating the accumulation of cementing substances at the surface of the pericarp. All these tissues are of maternal origin, which means that the hulled or naked caryopsis type solely depends on the mother plant genotype. In some of the T0 plants generated in the present study, the occurrence of both types of grains on the same spike was observed. This phenomenon can be explained by the limited penetrance of Cas9 nuclease activity causing chimeric primary transgenic plants in which induced mutations are confined to some sectors. Some of the cas9/gRNA-free T2 grains collected from homozygous T1 mutant plants also had still adherent hulls after standard mechanical threshing, yet in this case the hulls could readily be removed by hand, which was possible owing to the absence of the cementing layer between hull and pericarp. 
While previous work on NUD function relied on association studies [4-7], the present study adds unambiguous evidence for the causal effect of loss-of-function of the NUD gene on the formation of non-adherent hulls in barley. The results obtained also confirm the monogenic control of the caryopsis type in barley and demonstrate the possibility to convert hulled into naked barley by targeted mutagenesis of the NUD gene. Furthermore, it was demonstrated that the deletion of no more than a single nucleotide can be sufficient for this conversion.

\section{Pleiotropic effect of the nud locus}

Barley has high genetic diversity and comprises multiple combinations of different traits. Consequently, many differences have been observed between naked and hulled barley accessions. However, it is not always clear which of these phenotypes have just coincidentally occurred for instance owing to linkage drag, and which constitute truly causal consequences of a particular NUD allele. The most known differences between hulled and naked barley are related to the hull itself. Hulled barley has higher yield and higher crude fiber content. The hull normally constitutes $10-13 \%$ of the dry weight of barley grain [21] and the naked barley yields about $88 \%$ of hulled barley [22]. In a number of experiments aiming to establish the association between naked grain and other characteristics, contradictory results were obtained. A comparison of two hulled barleys and their naked mutant derivatives revealed only minor differences in yield and growth performance [23]. A further comparison of near-isogenic lines did also not establish that the naked phenotype be associated with any alterations as to amino acid composition of the grain [24], grains per $\mathrm{m}^{2}$, grains per spike, plant height, heading date and mildew resistance [22]. Crossings between naked and hulled Himalaya barleys revealed a multigenic nature of their differences [25] and again no evidence for pleiotropic effects of the nud locus, and in a similar study it was concluded that allelic variants of the NUD locus are unlikely to have an effect on seedling vigour [26]. Likewise, an analysis of doubled haploid lines derived from hybrids of hulled and naked barley did not reveal any pleiotropic effects of the naked phenotype on heading date, maturity, smut resistance, scald resistance, and spike density, but showed associations with lower plant density, reduced plant height as well as lower yield and grain weight [27]. However, the two latter effects may be well explained by the absence of the hull. A reduced number of plants grown per 100 viable grains was shown for naked barley accessions in comparison to hulled ones [28]. While Fusarium head blight severity was shown not to be different in hulled and naked barley, hulled barley accumulated significantly higher amounts of mycotoxins in the grain [29]. Taken together, these data as well as the successful cultivation of naked barley under different regional conditions suggest the absence of deleterious effects of the nud gene variant and at worst minor effects on important agronomic characteristics. It has been repeatedly reported that hulless barley has a potential for genetic improvement, and many disadvantages of naked varieties are not directly caused by the nud allele and thus may be overcome by site-directed mutagenesis using suitable hulled barley backgrounds.

\section{Potential of engineered nud knock-out lines for the advancement of naked barley breeding and the development of functional food}

The presence or absence of the hull is essential regarding the end use of barley grain. Rather than being processed for malting, the naked barley grain had been traditionally used for food in ancient times but was substituted by wheat in many regions including Europe. In the 20th and 21st centuries, the hulled barley has preferentially been cultivated in Europe and used mainly for malting, distilling and animal feed. Strong adhesion of lemma and palea to the pericarp is a major prerequisite of current brewing technologies [10]. However, as an important food source, the hulless barley is cultivated in Asia and northern Africa [30]. Nowadays, the interest in incorporating barley as a dietary component in food products is increasing because of the rising societal demand for food with potential health benefits. Barley contains high amounts of $\beta$-glucans. Humans do not have enzymes for $\beta$-glucan degradation, which is the reason that these molecules exert their beneficial properties as soluble fibers [31]. It was shown that barley $\beta$-glucan consumption is effective for the reduction of blood LDLcholesterol [32, 33] and reducing visceral fat obesity [34]. The effect of barley $\beta$-glucans on reducing the food glycemic index and their short-time blood sugar regulating properties is discussed [35-37]. In several studies, it was shown that naked barley has a higher content of $\beta$ glucans [38, 39] and the nud locus has been associated with a major QTL for this feature [40]. The effect of nud alleles on $\beta$-glucan content may be explained either by the closely linked location of the NUD gene and genes controlling $\beta$-glucan biosynthesis on the $7 \mathrm{H}$ chromosome or by a direct effect of the NUD gene on cell wall composition in the barley grain. The suppression of $\mathrm{cu}-$ ticle organization genes in hulled barley shown by comparative transcriptome analysis of two barley cultivars [11] and the presence of the cementing layer in hulled barley grain suggest differences in cell wall and cuticle organization in hulled and naked grain. This difference may have an effect on the nutritional value of the grain. The NUD/nud isogenic lines that are now available can be used to unambiguously confirm or falsify a causal 
relationship between naked caryopsis type and grain nutrient composition.

To use hulled barley for food, a pearling process is needed. Pearling removes the grain hull along with the surface layer of the grain which is particularly enriched with beneficial nutrients [21]. The use of naked barley for functional food is of particular health value because of optimal nutrient composition and the comparatively low costs of grain processing. The diversity of naked barley has been lost in regions where barley was no longer widely used for food. The introduction of non-adapted naked barley varieties in local breeding programs requires the genetic screening of germplasm collections and phenotyping under field conditions. This process does not allow for the development of dietary products containing naked barley in a short period of time. The possibility to convert current elite material to provide high-performance barley cultivars with naked grains can contribute to the provision of increasingly requested healthy food even beyond those regions where naked barley has been traditionally consumed. The developed and pre-tested highly efficient gRNA structures and genetic constructs for targeted knockout of the NUD gene may be directly used to convert advanced hulled germplasm into naked barley for dietary purposes.

\section{Conclusions}

The naked vs. hulled grain characteristic in barley is an example of a trait under monogenic control. The phenomenon of hull adhesion owing to the formation of a cementing layer is peculiar to barley, and there is no evidence in other species regarding WIN1/SHN1-like AP2/ERF factors controlling the formation of hulled or naked grains. The molecular function of the NUD transcription factor is still unknown and is not yet associated with any downstream genes or biochemical pathways. Using Cas endonuclease technology in the present study, a few barley lines with naked caryopses were created using the genetic background of the hulled cultivar Golden Promise. These lines can further be utilized as experimental material for reverse genetics studies on the molecular function of the NUD gene and its possible pleiotropic effects. The newly created NUD knockout lines may be of unprecedented utility for such investigations than previously available, chemically or X-rayinduced mutants or near-isogenic lines. The NUD genespecific gRNAs developed in the present study were demonstrated to be exceedingly efficient in inducing $\mathrm{KO}$ mutations in highly conserved target motifs and thus can be readily used for any transformable barley cultivar in order to convert it from hulled to naked grain type. This approach may be beneficial for the development of functional food in various global regions including those where naked barley has not been traditionally used as food.

\section{Methods \\ Guide RNA design and vector construction}

Target motif selection was performed using the online tools DESKGEN [41] and WU-CRISPR [42]. Guide-RNA secondary structures were modelled using the RNAfold tool (http://rna.tbi.univie.ac.at/cgi-bin/RNAWebSuite/RNAfold. cgi [38]). Off-target analysis was performed using the DESKGEN tool and via BLAST of target sequences at the IPK Barley BLAST Server (https://webblast.ipk-gatersleben. de/barley_ibsc/).

The nucleotide sequence of the NUD gene target fragment in the genome of barley cv. Golden Promise was confirmed by PCR with the primers Hv_Nud_F and Hv Nud_R2 (Additional file 5, Table S5) and Sanger sequencing. Four vectors harboring cas 9 and gRNA expression units were constructed on the basis of the pSH121 generic vector (Additional file 6, Figure S1) so as to address four target motifs within the HvNUD coding sequence (Fig. 1a). The vector construction was performed as described previously [15] using pairs of oligonucleotides listed in (Additional file 5: Table S5).

\section{Prevalidation of gRNA activity by ballistic DNA transfer to leaf epidermis cells}

To reveal the best performing gRNAs, a transient expression-based test for cleavage activity of Cas9/gRNA was performed as previously described [16] for each created cas9/gRNA construct. TARGET vectors were created using the generic vector pNB1 (GenBank: KU705395) as a backbone. The double-stranded oligonucleotides (Additional file 5, Table S5) corresponding to each target site with PAM were inserted in the pNB1 vector between the $B a m H I$ and EcoRI sites. In total, four TARGET vectors were created.

Leaf explants were taken from 10 days-old barley plants (cv. Golden Promise) grown in a glasshouse. Plasmid DNA was mixed using a total volume of $10 \mu \mathrm{l}$ in the following proportions: $7 \mu \mathrm{g}$ of target vector, $7 \mu \mathrm{g}$ of cas $9 /$ gRNA vector, $2 \mu \mathrm{g}$ of mCherry vector [16]. As positive control, a mixture of a pNB1 vector variant carrying an intact YFP expression cassette and mCherry vector was used, whereas a mixture of one of the target vectors and mCherry vector served as negative control. The coating of gold particles with plasmid DNA and bombardment were performed as previously described [16]. For each combination of constructs, two independent experiments were conducted, with two technical replicates per experiment (i.e. four in total). After bombardment, explants were kept in the dark for $28-48 \mathrm{~h}$. Reporter gene expression was observed with a Zeiss CLSM780 confocal laser scanning microscope using a $561 \mathrm{~nm}$ Helium- 
Neon-laser for mCherry detection (emission at $610 \mathrm{~nm}$ ) and a $488 \mathrm{~nm}$ Argon-laser for YFP detection (emission at $527 \mathrm{~nm}$ ). Activity of each cas $9 / \mathrm{gRNA}$ construct was deduced from the ratio between the number of cells accumulating both YFP and mCherry (YFP cells) and the number of transgenic cells (mCherry cells).

\section{Prevalidation of gRNA activity by protoplast transfection}

Leaf mesophyll protoplasts were isolated from etiolated barley seedlings (cv. Golden Promise) and co-transfected with one of the selected cas9/gRNA vectors and the GFP-expressing control vector pYF133 [43]. Transformed protoplasts were incubated in the dark at $21^{\circ} \mathrm{C}$ for 2 days. After determination of the portion of GFPpositive cells, genomic DNA was isolated from protoplasts and the target region was amplified using primers Hv_Nud_F4 - Hv_Nud_1exR (Additional file 5, Table S5). The mutation frequency and pattern in protoplasts were examined by deep amplicon sequencing. The proportion of GFP-positive cells was used to normalize the mutagenic activity of the respective cas $/$ gRNA vector. The non-parametric Kolmogorov-Smirnov test was used to compare the distributions of mutation types induced by selected gRNAs. Protoplast isolation, transformation, and amplicon sequencing were performed as described previously [15].

\section{Plant material}

The experiments of this investigation were conducted using the two-rowed spring-type barley (Hordeum vulgare L.) British cv. "Golden Promise" which is an X-ray mutant derived from its predecessor cv. "Maythorpe". The barley plants were cultivated as described previously [44].

\section{Binary vector construction and Agrobacterium-mediated barley transformation}

The cas 9 and selected gRNA expression units were transferred as $S f i$ fragments from the aforementioned pSH121-derived plasmids to the generic binary vector p6i-2x35s-TE9 (DNA Cloning Service, Hamburg, Germany), which resulted in the generation of the NUD-45 and NUD-50 transformation vectors. These vectors were transferred into $A$. tumefaciens strain AGL1 by electroporation. Agrobacterium-mediated gene transfer to immature embryos (barley cv. Golden Promise) was performed following a method described previously $[44,45]$. The primary transgenic plants were selected using $50 \mathrm{mg} / \mathrm{l}$ hygromycin and were then investigated by PCR for the presence of cas9 (Bie475 and zCas9-R1), gRNA (OsU3p-F1 and guide-specific RGEN Rev. Oligo) and progeny were additionally tested for the hygromycin phosphotransferase gene used as selectable marker (35S-F2 and HYG-R5). Primer structures are given in (Additional file 5, Table S5).

\section{Mutation detection in primary transgenic plants}

Mutations were detected by Sanger sequence data analysis. To this end, genomic DNA was extracted from candidate plants [46] and target and off-target regions were amplified and sequenced using the primers listed in Additional file 1, Table S5. Sanger chromatograms were analyzed for the presence of nucleotide sequence changes or abnormalities in the target motif.

\section{Genotyping and phenotyping of progeny}

Progeny of primary plants were growing for 1 month under standard glasshouse conditions $\left(16 \mathrm{~h}, 18^{\circ} \mathrm{C} / 8 \mathrm{~h}\right.$, $16^{\circ} \mathrm{C}$ ) and later transferred to speed breeding conditions $\left(22 \mathrm{~h}, 21^{\circ} \mathrm{C} / 2 \mathrm{~h}, 17^{\circ} \mathrm{C}\right)$ for 2.5 months. DNA was isolated from 1 week-old seedlings by using 96-well format DNA isolation [47]. Genotyping of target and off-target regions was done by PCR amplification and Sanger sequencing. After 3.5 months, fully matured spikes were harvested. As a standardized procedure, a hand thresher (Dreschhexe, Baumann Saatzuchbedarf, Germany) was used to remove the hulls from the spikes. For this, five spikes per line were threshed four times and grains were collected. Remaining hulls were removed by hand (Fig. $5 b)$.

\section{Supplementary information}

Supplementary information accompanies this paper at https://doi.org/10. 1186/s12870-020-02454-9.

Additional file 1: Supplementary Table S1. Guide RNA structure and activity in the transient expression test.

Additional file 2: Supplementary Table S2. Evaluation of protoplast transfection efficiency.

Additional file 3: Supplementary Table S3. Deep-sequencing results for target motifs in mutated protoplast population.

Additional file 4: Supplementary Table S4. Target mutations in T0 and $\mathrm{T} 1$ detected in selected plants.

Additional file 5: Supplementary Table S5. List of primers and oligonucleotides used in this study.

Additional file 6: Supplementary Figure S1. Sequence data and map of generic vector pSH121

\section{Abbreviations}

ERF: Ethylene Response Factor; KO: Knockout; LDL-cholesterol: Low-density lipoprotein cholesterol; PAM: Protospacer-adjacent motif; QTL: Quantitative trait locus; WIN1/SHN1: Wax Inducer 1/Shine 1

\section{Acknowledgements}

We thank Vasily Koval (ICG Novosibirsk) for making and providing a photo of barley grains (used in Fig. 3). The excellent technical assistance of Sibylle Freist, Sabine Sommerfeld and Nicole Schäfer (IPK Gatersleben) is gratefully acknowledged.

\section{About this supplement}

This article has been published as part of BMC Plant Biology Volume 20 Supplement 1, 2020: Selected articles from the 5th International Scientific Conference "Plant genetics, genomics, bioinformatics, and biotechnology" (PlantGen2019). The full contents of the supplement are available online at https://bmcplantbiol.biomedcentral.com/articles/supplements/volume-20supplement-1. 


\section{Authors' contributions}

SVG and $\mathrm{CH}$ designed the experiments, assembled and tested the genetic constructs, and performed genotyping and phenotyping of mutant plants; JK and EKK conceived and designed the project and the experimental strategy; EVK and AMK contributed to the experimental design and to the plant genotyping; IO contributed to protoplast isolation and transfection; $\mathrm{SH}$ designed and constructed the generic vector for site-directed genome modification; AVK contributed to the experimental design and manuscript revision; SVG, JK, CH and EKK wrote and revised the manuscript. All authors have read and approved the final manuscript.

\section{Funding}

The study is supported by the Russian Science Foundation, project No. 1614-00086. Publication costs are funded by the Russian Science Foundation, project No. 16-14-00086. S.V.G. is grateful to Kurchatov Genomics Center of IC\&G for support. Mutant barley lines were cultivated in IC\&G plant growth facility (supported by project 0324-2019-0039). The funding bodies played no role in the experimental design, the data collection, analysis and interpretation and in writing the manuscript.

\section{Availability of data and materials}

All data generated or analyzed during this study are included in this published article and its supplementary materials file or are freely accessible in a data repository (plasmid pNB1: GenBank accession KU705395). Materials generated in this study are available from the corresponding authors upon reasonable request.

\section{Ethics approval and consent to participate}

Not applicable.

\section{Consent for publication}

Not applicable.

\section{Competing interests}

The authors declare no conflict of interest.

\begin{abstract}
Author details
${ }^{1}$ Institute of Cytology and Genetics, SB RAS, Novosibirsk 630090, Russia. ${ }^{2}$ Novosibirsk State University, Novosibirsk 630090, Russia. ${ }^{3}$ Kurchatov Genomics Center, Institute of Cytology and Genetics, SB RAS, Novosibirsk 630090, Russia. ${ }^{4}$ Leibniz Institute of Plant Genetics and Crop Plant Research (IPK), 06466 Gatersleben, Germany. ${ }^{5}$ Vavilov Institute of Plant Genetic Resources (VIR), Saint Petersburg 190000, Russia.
\end{abstract}

\section{Received: 29 January 2020 Accepted: 6 March 2020}

Published: 14 October 2020

\section{References}

1. Zohary D, Weiss E, Hopf M. Current state of the art. In: Domestication of Plants in the Old World: Oxford University Press; 2012. p. 1-8. https://doi. org/10.1093/acprof:osobl/9780199549061.003.0001.

2. Helbaek H. Domestication of food plants in the Old World. Sci New Ser. 1959;130:365-72.

3. Taketa S, Kikuchi S, Awayama T, Yamamoto S, Ichii M, Kawasaki S Monophyletic origin of naked barley inferred from molecular analyses of a marker closely linked to the naked caryopsis gene (nud). Theor Appl Genet. 2004;108:1236-42. https://doi.org/10.1007/s00122-003-1560-1.

4. Zeng X, Guo Y, Xu Q, Mascher M, Guo G, Li S, et al. Origin and evolution of qingke barley in Tibet. Nat Commun. 2018;9:5433. https://doi.org/10.1038/ s41467-018-07920-5.

5. Taketa S, Amano S, Tsujino Y, Sato T, Saisho D, Kakeda K, et al. Barley grain with adhering hulls is controlled by an ERF family transcription factor gene regulating a lipid biosynthesis pathway. Proc Natl Acad Sci. 2008;105:40627. https://doi.org/10.1073/pnas.0711034105.

6. Yu S, Long H, Deng G, Pan Z, Liang J, Zeng X, et al. A single nucleotide polymorphism of nud converts the caryopsis type of barley (Hordeum vulgare L.). Plant Mol Biol Report. 2016;34:242-8. https://doi.org/10.1007/ s11105-015-0911-9.

7. Wabila C, Neumann K, Kilian B, Radchuk V, Graner A. A tiered approach to genome-wide association analysis for the adherence of hulls to the caryopsis of barley seeds reveals footprints of selection. BMC Plant Biol. 2019;19:95. https://doi.org/10.1186/s12870-019-1694-1.

8. Gasparis S, Kała M, Przyborowski M, Łyżnik LA, Orczyk W, Nadolska-Orczyk A. A simple and efficient CRISPR/Cas9 platform for induction of single and multiple, heritable mutations in barley (Hordeum vulgare L.). Plant Methods. 2018;14:111. https://doi.org/10.1186/s13007-018-0382-8.

9. Salamini F, Özkan H, Brandolini A, Schäfer-Pregl R, Martin W. Genetics and geography of wild cereal domestication in the near east. Nat Rev Genet. 2002;3:429-41. https://doi.org/10.1038/nrg817.

10. Hoad SP, Brennan M, Wilson GW, Cochrane PM. Hull to caryopsis adhesion and grain skinning in malting barley: identification of key growth stages in the adhesion process. J Cereal Sci. 2016;68:8-15. https://doi.org/10.1016/j.jcs. 2015.10.007.

11. Duan $\mathrm{R}$, Xiong H, Wang A, Chen G. Molecular mechanisms underlying hullcaryopsis adhesion/separation revealed by comparative transcriptomic analysis of covered/naked barley (Hordeum vulgare L.). Int J Mol Sci. 2015;16: 14181-93.

12. Kumlehn J, Pietralla J, Hensel G, Pacher M, Puchta H. The CRISPR/Cas revolution continues: from efficient gene editing for crop breeding to plant synthetic biology. J Integr Plant Biol. 2018;60:1127-53. https://doi.org/10. 1111/jipb.12734

13. Korotkova AM, Gerasimova SV, Khlestkina EK. Current achievements in modifying crop genes using CRISPR/Cas system. Vavilov J Genet Breed. 2019;23:29-37. https://doi.org/10.18699/NJ19.458.

14. Koeppel I, Hertig C, Hoffie R, Kumlehn J. Cas endonuclease technology-a quantum leap in the advancement of barley and wheat genetic engineering. Int J Mol Sci. 2019;20:2647. https://doi.org/10.3390/ ijms20112647.

15. Gerasimova SV, Korotkova AM, Hertig C, Hiekel S, Hoffie R, Budhagatapalli N, et al. Targeted genome modifcation in protoplasts of a highly regenerable Siberian barley cultivar using RNA-guided Cas9 endonuclease. Vavilov J Genet Breed. 2019;22:1033-9. https://doi.org/10.18699/NJ18.447.

16. Budhagatapalli N, Schedel S, Gurushidze M, Pencs S, Hiekel S, Rutten T, et al. A simple test for the cleavage activity of customized endonucleases in plants. Plant Methods. 2016;12:18. https://doi.org/10.1186/s13007-016-01186.

17. Beier S, Ulpinnis C, Schwalbe M, Münch T, Hoffie R, Koeppel I, et al. Kmasker plants - a tool for assessing complex sequence space in plant species. Plant J. 2020:tpj.14645. https://doi.org/10.1111/tpj.14645.

18. Kapusi E, Corcuera-Gómez M, Melnik S, Stoger E. Heritable genomic fragment deletions and small indels in the putative engase gene induced by CRISPR/Cas9 in barley. Front Plant Sci. 2017;8:540. https://doi.org/10. 3389/fpls.2017.00540.

19. Holubová K, Hensel G, Vojta P, Tarkowski P, Bergougnoux V, Galuszka P. Modification of barley plant productivity through regulation of cytokinin content by reverse-genetics approaches. Front Plant Sci. 2018;9:1676. https://doi.org/10.3389/fpls.2018.01676.

20. Li M, Hensel G, Mascher M, Melzer M, Budhagatapalli N, Rutten T, et al. Leaf variegation and impaired chloroplast development caused by a truncated CCT domain gene in albostrians barley. Plant Cell. 2019;31:1430-45. https:// doi.org/10.1105/tpc.19.00132.

21. Bleidere M, Jansone Z, Grunte I, Jakobsone I. Biochemical composition of spring barley grain pearled to varying degrees. Proc Latv Acad Sci Sect B Nat Exact Appl Sci. 2017;71:468-73. https://doi.org/10.1515/prolas-20170082.

22. Barabaschi D, Francia E, Tondelli A, Gianinetti A, Stanca AM, Pecchioni N. Effect of the nud gene on grain yield in barley. Czech J Genet Plant Breed. 2012;48(1):10-22. https://doi.org/10.17221/117/2011-CJGPB.

23. Takahashi $\mathrm{R}$, Inamura $\mathrm{H}$, Matsumoto $\mathrm{T}$. Effects of the genes for covered and naked kernels on some agronomic characters in barley. Ber Ohara Inst Indw Biol Okayama Univ. 1962;11:385-92 http://eprints.lib.okayama-u.ac.jp/en/4 9890

24. Bhatty RS. The potential of hull-less barley. Cereal Chem. 1986;63:97-103.

25. Witcombe JR, Murphy PJ. Covered and naked barleys from the Himalaya. Theor Appl Genet. 1986;71:736-41. https://doi.org/10.1007/BF00263272.

26. Capo-chichi L, Kenward K, Nyachiro J, Anyia A. Nud locus and the effects on seedling vigour related traits for genetic improvement of hulless barley. J Plant Sci Mol Breed. 2012;1:2. https://doi.org/10.7243/2050-2389-1-2.

27. Choo T-M, Ho KM, Martin RA. Genetic analysis of a hulless $x$ covered cross of barley using doubled-haploid lines. Crop Sci. 2001:41:1021. https://doi. org/10.2135/cropsci2001.4141021x. 
28. Dickin E, Steele K, Edwards-Jones G, Wright D. Agronomic diversity of naked barley (Hordeum vulgare L.): a potential resource for breeding new food barley for Europe. Euphytica. 2012;184:85-99. https://doi.org/10.1007/ s10681-011-0567-y.

29. Legzdina L, Buerstmayr H. Comparison of infection with Fusarium head blight and accumulation of mycotoxins in grain of hulless and covered barley. J Cereal Sci. 2004;40:61-7. https://doi.org/10.1016/j.jcs.2004.03.002.

30. Baik B-K, Ullrich SE. Barley for food: characteristics, improvement, and renewed interest. J Cereal Sci. 2008;48:233-42. https://doi.org/10.1016/j.jcs. 2008.02.002.

31. Kinner M, Nitschko S, Sommeregger J, Petrasch A, Linsberger-Martin G Grausgruber H. Naked barley-optimized recipe for pure barley bread with sufficient beta-glucan according to the EFSA health claims. J Cereal Sci. 2011;53:225-30. https://doi.org/10.1016/j.jcs.2011.01.001.

32. Tiwari U, Cummins E. Meta-analysis of the effect of $\beta$-glucan intake on blood cholesterol and glucose levels. Nutrition. 2011;27:1008-16. https://doi. org/10.1016/j.nut.2010.11.006

33. Zhu X, Sun X, Wang M, Zhang C, Cao Y, Mo G, et al. Quantitative assessment of the effects of beta-glucan consumption on serum lipid profile and glucose level in hypercholesterolemic subjects. Nutr Metab Cardiovasc Dis. 2015;25: 714-23. https://doi.org/10.1016/.numecd.2015.04.008.

34. Aoe S, Ichinose Y, Kohyama N, Komae K, Takahashi A, Abe D, et al. Effects of high $\beta$-glucan barley on visceral fat obesity in Japanese individuals: a randomized, double-blind study. Nutrition. 2017;42:1-6. https://doi.org/10. 1016/.nut.2017.05.002.

35. AbuMweis S, Thandapilly SJ, Storsley J, Ames N. Effect of barley $\beta$-glucan on postprandial glycaemic response in the healthy human population: a metaanalysis of randomized controlled trials. J Funct Foods. 2016;27:329-42. https://doi.org/10.1016/j.jf.2016.08.057.

36. Nilsson AC, Östman EM, Knudsen KEB, Holst JJ, Björck IME. A cereal-based evening meal rich in indigestible carbohydrates increases plasma butyrate the next morning. J Nutr. 2010;140:1932-6.

37. Thondre PS, Henry CJK. High-molecular-weight barley $\beta$-glucan in chapatis (unleavened Indian flatbread) lowers glycemic index. Nutr Res. 2009;29:4806. https://doi.org/10.1016/.nutres.2009.07.003.

38. Gruber AR, Lorenz R, Bernhart SH, Neubock R, Hofacker IL. The Vienna RNA websuite. Nucleic Acids Res. 2008;36:W70-4. https:/doi.org/10.1093/nar/gkn188.

39. Wirkijowska A, Rzedzicki Z, Zarzycki P, Sobota A, Sykut-doma E. Chemical composition of naked barley for production of functional food. Acta Agrophysica. 2016;23:287-301.

40. Mežaka I, Legzdina L, Bleidere M, Rostoks N. Whole genome association mapping identifies naked grain locus NUD as determinant of $\beta$-glucan content in barley. Zemdirbyste. 2011;98:283-92.

41. Doench JG, Hartenian E, Graham DB, Tothova Z, Hegde M, Smith I, et al. Rational design of highly active sgRNAs for CRISPR-Cas9-mediated gene inactivation. Nat Biotechnol. 2014;32:1262-7. https://doi.org/10.1038/nbt.3026

42. Wong N, Liu W, Wang X. WU-CRISPR: characteristics of functional guide RNAs for the CRISPR/Cas9 system. Genome Biol. 2015;16:218. https://doi.org/ 10.1186/s13059-015-0784-0.

43. Fang Y, Akula C, Altpeter F. Agrobacterium-mediated barley (Hordeum vulgare $\mathrm{L}$.) transformation using green fluorescent protein as a visual marker and sequence analysis of the T-DNA $\propto$ barley genomic DNA junctions. J Plant Physiol. 2002;159:1131-8. https://doi.org/10.1078/0176-1617-00707.

44. Hensel G, Valkov V, Middlefell-Williams J, Kumlehn J. Efficient generation of transgenic barley: the way forward to modulate plant-microbe interactions. J Plant Physiol. 2008;165:71-82. https://doi.org/10.1016/j.jplph.2007.06.015.

45. Marthe C, Kumlehn J, Hensel G. In: Wang K, editor. Barley (Hordeum vulgare L.) transformation using immature embryos, vol. 1: Agrobacterium Protocols, Methods in Molecular Biology; 2015. p. 71-83. https://doi.org/10.1007/978-14939-1695-5_6.

46. Pallotta MA, Graham RD, Langridge P, Sparrow DHB, Barker SJ. RFLP mapping of manganese efficiency in barley. Theor Appl Genet. 2000;101: 1100-8. https://doi.org/10.1007/s001220051585.

47. Stein N, Herren G, Keller B. A new DNA extraction method for highthroughput marker analysis in a large-genome species such as Triticum aestivum. Plant Breed. 2001;120:354-6. https://doi.org/10.1046/j.1439-0523. 2001.00615.x.

\section{Publisher's Note}

Springer Nature remains neutral with regard to jurisdictional claims in published maps and institutional affiliations.

Ready to submit your research? Choose BMC and benefit from:

- fast, convenient online submission

- thorough peer review by experienced researchers in your field

- rapid publication on acceptance

- support for research data, including large and complex data types

- gold Open Access which fosters wider collaboration and increased citations

- maximum visibility for your research: over $100 \mathrm{M}$ website views per year

At BMC, research is always in progress.

Learn more biomedcentral.com/submissions 